$$
\text { punto org }
$$

Collana diretta da Luigi Maria Sicca

79 


\title{
Paola Ventrella
}

\section{LUDEBAT BELLEROFONTES}

Vita $\mathcal{E}$ Opere di Bellerofonte Castaldi, liutista modenese

\author{
Prefazione \\ Franco Pavan \\ Postfazione \\ Diego Cantalupi
}

Editoriale Scientifica

Napoli 


\section{punt ○org}

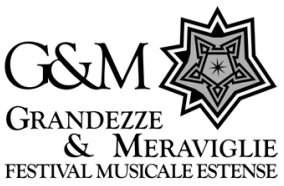

(c) Copyright 2020 Editoriale Scientifica s.r.l.

Via San Biagio dei Librai, 39 - 80138 Napoli

www.editorialescientifica.com info@editorialescientifica.com

ISBN 978-88-9391-981-4 


\section{Indice}

Il Racconto de li capitoli che sono in questo libro

9 Nota editoriale

Luigi Maria Sicca

11 Prefazione

Franco Pavan

15 Premessa

17 1. STATO DELL'ARTE

21 2. La poesia di CASTALdi

29 3. Prologo

31 4. La Biografia attraverso le Rime

63 5. La Corte e l'Accademia

83 6. CAPRicCI A DUE STROMENTI

103 7. Il Manoscritto G.239

$104 \quad 7.1$ Descrizione Fisica

$107 \quad 7.2$ Stato attuale degli studi

$113 \quad 7.3$ Le concordanze

$123 \quad 7.4$ Studio Calligrafico

1297.5 Filigrane

$134 \quad 7.6$ Conclusioni 
137 8. Appendice

141 Postfazione

Diego Cantalupi

145 Bibliografia

153 Indice dei nomi

157 Ringraziamenti

159 Hanno scritto nella Collana punto org 
Ad Anna Maria, che ha ispirato ogni passo di questo percorso 

Accogliamo con piacere nella Collana punto org il volume di Paola Ventrella «"Ludebat Bellerofontes". Vita E Opere di Bellerofonte Castaldi, liutista modenese», grazie al contributo concesso dalla Direzione generale Educazione, ricerca e istituti culturali del Ministero per i beni e le attività culturali e per il turismo.

Mi piace da subito evidenziare la costruttiva collaborazione con "Grandezze \& Meraviglie - Festival Musicale Estense", il più longevo nel campo della musica antica della Regione Emilia-Romagna, una realtà fra le più innovative e articolate nel suo genere non solo in Italia, ma anche a livello internazionale, dedicata al repertorio antico (dal Medioevo al primo Ottocento), forte del rigore che deriva dalla pratica del suono attraverso strumenti e prassi esecutive dell'epoca.

Il libro vede la luce nell'ambito delle attività della Associazione di ricerca internazionale puntOorg che attribuisce ai saperi musicali (declinati ad amplio spettro e, al contempo, con la profondità "scientifica" che caratterizza qualunque approccio intra-disciplinare che voglia dirsi solido) e alla cultura classica il ruolo di fondamenta della conoscenza, per leggere e interpretare i tempi odierni e le complesse dimensioni della convivenza che caratterizzano questa contemporaneità che ci è capitata in sorte.

Già in precedenti occasioni abbiamo proposto un così delicato intreccio inter-disciplinare nella convinzione che musica, filosofia, archivistica, economia, scienze umane e sociali e scienze dure debbano (ancor prima che possano, viste anche le urgenze planetarie del complicato 2020 in corso) imparare a dialogare in modo paritario intorno a una tavola rotonda (senza quindi capotavola disegnato a priori), perché è così - per noi di puntOorg - che 
sarà possibile costruire migliori scenari dello "stare-insieme", contenitivi e protettivi, al riparo da percorsi di crescita economic-istici, laddove ogni ismo rischia di esitare in dittature, prima di pensiero e poi di azione e interazione e corrispettiva, probabile, ribellione da parte della Natura.

Il lavoro di Paola Ventrella si presenta dunque come una nuova perla preziosa che si aggiunge a questa epistemologia sfidante, interculturale e pronta alle forme più rigorose dell'ibridazione accademica e scientifica, cui anche le moderne organizzazioni aziendali - che assorbono la maggior parte del tempo della nostra vita adulta e produttiva (oramai anche, talvolta, post-produttiva) - potranno beneficiare.

Luigi Maria Sicca Napoli, Università degli Studi Federico II 



\section{Hanno scritto nella Collana punto org}

www.puntoorg.net

1. L.M. Sicca (a cura di)', Leggere e scrivere organizzazioni. Estetica, umanesimo e conoscenze manageriali (con postfazione di F. Piro), 2010.

2. L.M. Sicca, Alla fonte dei saperi manageriali. Il ruolo della musica nella ricerca per l'innovazione e per la formazione delle risorse umane, 2012.

3. A. Di Scipio, Pensare le tecnologie del suono e della musica (con prefazione di R. Diana), 2012.

4. R. Musto, Scienza Natura Cambiamento (con prefazione di M. Nicode$\mathrm{mi}), 2012$.

5. R. Musto, Novalis. L'assoluto e le cose (con prefazione di C. Albarella e postfazione di G. Imbruglia), 2013.

6. Aa.Vv.", I linguaggi dell'organizzare. Musica e testo tra dono e disinteresse, 2013.

7. B. Masiello, Fiducia nelle reti. Strategie per la crescita nei mercati internazionali delle PMI (con prefazione di F. Izzo), 2013.

8. Aa.Vv. ${ }^{\text {II }}$, Tavola rotonda. Umanesimo del management attraverso gli occhi dell'altro, 2013.

9. M. Calcagno, Narrare terre di mezzo. Management arte design (con prefazione di S. Faccipieri e postfazione di A. Comacchio), 2013.

10. R. Diana, Disappartenenza dell'To. Filosofia e musica verso Samuel Beckett (con prefazione di L.M. Sicca), 2014.

${ }^{1}$ Con scritti di Per Olof Berg e Kristian Kreiner, Robert W. Witkin, Barbara Czarniawska e Carl Rhodes, Ken Starkey e Sue Tempest, John Hendry, Karin Knorr Cetina.

" Con scritti di Luigi Maria Sicca, Umberto di Porzio, Rosario Diana, Agostino Di Scipio, Mariella De Simone, Bernardo Maria Sannino, Chiara Mallozzi, Lorenzo Pone, Giancarlo Turaccio.

"I Con scritti di Luigi Maria Sicca, Francesco Izzo, Maura Striano, Giulia Dell'Aquila, Felice Casucci, Francesco Perillo, Rosario Diana, Paola Giampaolo, Davide Bizjak, Gilberto-Antonio Marselli, Franco Vitelli, Maria Rosaria Napolitano. 
11. Aa.Vv. ${ }^{\mathrm{IV}}$, Sergio Piro. Maestri e allievi, 2014.

12. F.D. Perillo (a cura di) ${ }^{\mathrm{v}}$, Impresa imperfetta, 2014.

13. L.M. Sicca, L. Zan (a cura di) ${ }^{\mathrm{v}}$, Management Arti Culture. Resoconto del primo anno del GSA - Accademia Italiana di Economia Aziendale, 2014.

14. M. Iaccarino, Un mondo assetato. Come il bisogno di acqua plasma la civiltà (con prefazione di F.P. Casavola e postfazione di A. Giannola), 2015.

15. F. Piro, Manuale di educazione al pensiero critico. Comprendere e argomentare (con prefazione di T. De Mauro), 2015.

16. F. D'Errico, Fuor di metafora. Sette osservazioni sull'improvvisazione musicale (con prefazione di P. de Vita e postfazione di M. Maldonato), 2015.

17. E. Mollona, Computer Simulation in Social Sciences. A Logic of Enquiry (with a preface by L.M. Sicca, a foreword by G. Colombo and an afterword by D. Secchi), 2015.

18.S. Oliverio, L.M. Sicca, P. Valerio ${ }^{\mathrm{vI}}$, Transformare le pratiche nelle organizzazioni di lavoro e di pensiero (con prefazione di G. Manfredi), 2015.

19. P. Valerio, C. Bertolazzi, P. Marcasciano (a cura di) ${ }^{\mathrm{v} I I}$, Transformare l'organizzazione dei luoghi di detenzione. Persone transgender e gender nonconforming tra diritti e identità (con prefazione di L.M. Sicca), 2016.

Iv Con scritti di Giuseppe Cantillo, Tullio De Mauro, Aldo Masullo, Mariapaola Fimiani, Teresa Capacchione, Antonio Mancini, Roberto Beneduce, Enrico De Notaris, Fulvio Marone, Dario Stefano Dell'aquila, Luigi Maria Sicca, Francesco Piro.

v Con scritti di Pier Luigi Celli, Eugenio Mazzarella, Enzo Rullani, Luigi Maria Sicca, Francesco Varanini.

vi Con scritti di Stefano Baia Curioni, Paola Dubinie Ludovica Leone, Sara Bonini Baraldi e Luca Zan, Monica Calcagno e Luigi M. Sicca, Donata Collodi, Francesco Crisci e Andrea Moretti, Roberto Ferrari e Alessandro Hinna, Francesco Giaccari, Francesca Imperiale e Valentina Terlizzi, Daniele Goldoni, Pamela Palmi.

vi Con scritti di Anna Lisa Amodeo, Christian Ballarin, Davide Bizjak, Ilaria Boncori e Paolo Fazzari, Rossella Bonito Oliva, Simone Cangelosi, Marco De Giorgi, Guglielmo Faldetta, Vittoria Fiorelli, Stefano Maltese, Porpora Marcasciano, Piergiorgio Masi, Antonia Monopoli e Chiara Repetto, Andrea Morniroli, Edoardo Mollona, Cristiano Scandurra, Luca Solari, Maria Spanò, Maria Gigliola Toniollo.

viI Con scritti di: Paolo Valerio, Giuseppe Ferraro, Carmen Bertolazzi, Alexander Hochdorn, Porpora Marcasciano, Luca Chianura, Damiana Massara, Daniela A. Nadalin, Adriana Godano, Luca Chianura, Vittoria Colonna, Elia De Caro, Tito Flagella, Anna Lorenzetti. 
20. M.R. Napolitano, V. Marino (a cura di) ${ }^{\mathrm{Ix}}$, Cultural Heritage e Made In Italy. Casi ed esperienze di marketing internazionale (con prefazione di G. Volpe e postfazione di A. Mattiacci), 2016.

21. M. Lusiani, Discourses of Planning (with a preface by L. Zan and an afterword by A. Langley), 2016.

22. F.D. Perillo, Simposio manageriale (con prefazione di A. Masullo e postfazio-ne di P.L. Celli), 2016.

23. P. Ferri, I commissariamenti nel settore culturale italiano. Obiettivi, azioni, risultati (con prefazione di L. Zan e postfazione di G. Grossi), 2016.

24. L. Pareschi, Controcampo letterario. Strategie di intermediazione e accesso all'industria editoriale (con prefazione di P. Dubini e postfazione di G. Colombo), 2016.

25. G.-A. Marselli, Mondo contadino e azione meridionalista. L'esperienza del Gruppo Rossi-Doria a Portici (con prefazione di E. Mazzetti e postfazione di F. Vitelli), 2016.

26. F. Accardix, Risk and Control Governance. A value-creation perspective (with a preface by A. De Nicola; an introduction by V. Atella and an afterword by S. Bozzolan), 2017.

27. I. Boncori (ed.) $)^{x 1}, L G B T+$ Perspectives. The University of Essex Reader, (with a foreword by A. Forster), 2017.

28. A. Papa, “... Una cappella cavata dentro il monte...". Storia minima del complesso monastico di S. Lucia al Monte (con prefazione di L. d'Alessandro), 2017.

זx Con scritti di Loretta Battaglia, Giuseppe Bertoli, Roberta Biandolino, Michelle Bonera, Enrico Bonetti, Mauro Cavallone, Elena Cedrola, Marta Cerquetti, Maria Chiarvesio, Anna Codini, Emanuela Conti, Eleonora Di Maria, Barbara Francioni, Antonella Garofano, Francesco Izzo, Giulia Lanzilli, Gaetano Macario, Giulio Maggiore, Francesca Magno, Vittoria Marino, Barbara Masiello, Michela Matarazzo, Alberto Mattiacci, Marta Maria Montella, Fabio Musso, Maria Rosaria Napolitano, Alessandro Pagano, Tonino Pencarelli, Giovanna Pegan, Michele Quintano, Riccardo Resciniti, Marcello Risitano, Angelo Riviezzo, Savino Santovito, Elisabetta Savelli, Michele Simoni, Annarita Sorrentino, Raffaella Tabacco, Donata Vianelli.

${ }^{x}$ Con scritti di Roberto Rosato, Nicoletta Mincato, Carlo Nicoletti, Paolo De Paolis, Alessandro Salibra Bove.

хл Con scritti di Alison J. Taylor-Lamb, Jamie Raines, Thomas Currid and Carl Chandra, Martin Harrison and Peter Martin, Rainer Shulze, Fleur Jeans and Teresa Eade, Tuesday Wats, Amy Anderson, Sco Lawley. 
29. R. Diana, L.M. Sicca, G. Turaccio ${ }^{\mathrm{xII}}$, Risonanze. Organizzazione, musica, scienze (con prefazione di A. Strati e postfazione di A. Solbiati), 2017.

30. F. D'Errico, Armonia funzionale e modalità. Rudimenti per l'improvvisazione a indirizzo jazzistico (con introduzione di F. Piro e prefazione di R. Grisley), 2017.

31. M. Calcagno, Interpreting Innovation. Design Creativity Art (with a foreword by F. Izzo; preface by A. Moretti and an afterword by J. Metelmann), 2017.

32. G. Balirano, Gardai \& Badfellas: The Discursive Construction of Organised Crime in the Irish Media (with a foreword by L.M. Sicca), 2017.

33. M.C. Mason, A. Moretti, Tattoo Management. Mercati, attori, valore, 2017.

34. P. Testa, Innovazione del modello di business. Le dimensioni latenti nella letteratura di management (con prefazione di L. Cantone), 2017.

35. L. Massa, Viva 'o re! Municipio e dintorni (con introduzione di L.M. Sicca; prefazione di E. Borgonovi e postfazione di C. Mochi Sismondi), 2017.

36. F. Pavan, Memini. Piccole storie di storia della musica (con introduzione di E. Mazzarella; prefazione di R. Alessandrini e postfazione di V. Moroni), 2017.

37. C. Mallozzi, D. Tortora ${ }^{\mathrm{xII}}$, La bottega del suono. Mario Bertoncini. Maestri e allievi (con prefazione di M. Nicodemi e postfazione di L.M. Sicca), 2017.

38. G. Melis, Collaborazione e apprendimento nei processi di co-creazione di valore. Il caso delle destinazioni turistiche (con prefazione di M.R. Napolitano e postfazione di B. Argiolas), 2018.

xII Con scritti di Davide Bizjak, Dario Casillo, Rosario Diana, Umberto Di Porzio, Agostino Di Scipio, Chiara Mallozzi, Mario Nicodemi, Lorenzo Pone, Rosalba Quindici, Sonia Ritondale, Tommaso Rossi, Bernardo Maria Sannino, Luigi Maria Sicca, Cristian Sommaiuolo, Giancarlo Turaccio, Paolo Valerio.

xIII Con scritti di Mario Bertoncini, Davide Bizjak, Gianmario Borio, Pietro Cavallotti, Andrew Culver, Francesco D'Errico, Charles de Mestral, Michelangelo Lupone, Chiara Mallozzi, Alessandro Mastropietro, Mario Nicodemi, Luigino Pizzaleo, Lorenzo Pone, Ingrid Pustijanac, John Rea, Bernardo Maria Sannino, Luigi Maria Sicca, Daniela Tortora. 
39. G. Viglia, A.C. Invernizzi, Il ruolo dell'hubris nella gestione imprenditoriale (con prefazione di C. Mauri), 2018.

40. T. Russo Spena, C. Mele, Practising innovation. A Sociomaterial View (with a foreword by E. Gummesson; preface by J. Spohrer and an afterword by P. Stampacchia), 2018.

41. I. Boncori, Race, Ethnicity and Inclusion. The University of Essex Reader (with a foreword by A. Forster and a postface by M. Śliwa), 2018.

42. K.E. Russo, The Evaluation of Risk in Institutional and Newspaper Discourse. The Case of Climate Change and Migration (with a preface by G. Bettini), 2018.

43. R. Pera, When Consumers get Creative. Cocreation in the Individual and Collective Realm (with a preface by D. Dalli), 2018.

44. F. Piro, L.M. Sicca, P. Maturi, M. Squillante, M. Striano (a cura di) ${ }^{\mathrm{xiv}}$, Sfide didattiche. Il pensiero critico nella scuola e nell'università (con prefazione di F. Sabatini), 2018.

45. R. Quaglia, Bravi ma basta! Su certe premesse, promesse e catastrofi culturali (con introduzione di L.M. Sicca; prefazione di J. Mills e postfazione di F. Barca), 2018.

46. B. Czarniawska, La narrazione nelle scienze sociali, I edizione italiana a cura di L.M. Sicca, F. Piro, I. Boncori, 2018.

47. F. Longobardi, Le affinità del lessico, 2018.

48. G. Calogero, L'abbiccì della democrazia. E altri scritti, a cura di R. Trombelli, con una testimonianza di G. Sasso, 2018.

49. V. Fiorelli (a cura di $)^{\mathrm{xv}}$, Margini e confini. Attraversamenti di metodi e linguaggi tra comunicazione, didattica e possibilità della ricerca (con prefazione di L. d'Alessandro), 2018.

50. G. Cundari, Il mondo: una bella prigione? Riflessioni geografiche, 2018.

xiv Con scritti di Maura Striano, Rosaria Capobianco e Maria Rita Petitti, Francesco Piro, Roberta Gimigliano, Monica Mollo, Gerarda Fattoruso, Maria Incoronata Fredella, Maria Grazia Olivieri, Massimo Squillante e Antonia Travaglione, Pietro Maturi, Fabio Maria Risolo, Luca Marano, Luigi Maria Sicca, Giuseppe Recinto, Mario Nicodemi, Chiara Mallozzi e Luigi Marolda, Luigi Proserpio, Davide Bizjak, Paolo Canonico, Stefano Consiglio, Ernesto De Nito e Teresa Anna Rita Gentile, Natascia Villani.

xv Con scritti di Giuliano Amato, Gianluca Bocchi, Massimo Abdallah Cozzolino, Diego Davide, Lucia Donsi, Amedeo Feniello, Vittoria Fiorelli, Luigi Manconi, Roberta Morosini, Gianmarco Pisa, Ciro Pizzo, Leopoldo Repola, Stefano Rodotà, Francesco Varanini. 
51. M. Nicotra, Il canto dei sireni. Invenzioni trans/singolari e psicoanalisi lacaniana (con prefazione di A. Vicens e postfazione di P. Guazzo), 2019.

52. L. Marano, Come parlano i giovani. Una indagine di sociolinguistica urbana (con prefazione di P. Maturi), 2019.

53. M. Floris, R. Tronci, C. Dessi, A. Dettori, Imprese familiari e imprenditorialità. La sfida del cambiamento (con prefazione di F. Cabiddu), 2019.

54. T.T. Lennerfors, L. Mitchell (eds) ${ }^{\mathrm{xv}}$, SCOS. Searching Collectively for Our Soul, 2019.

55. Aa.Vv. ${ }^{\text {xvII }}$, Lucio Sicca. Maestri e allievi, 2019.

56. E. Sacerdote ${ }^{\mathrm{x} \amalg}$, Breviario sul pensiero strategico. Discorsi e percorsi per conquistare il futuro migliore, 2019.

57. M.E. Santagati, L. Zan (a cura di) ${ }^{\mathrm{xix}}$, Imprenditorialità e settore museale. Esperienze e prospettive di cambiamento, 2019.

58. F.D. Perillo, D. Bizjak, L'impresa nello zaino. La Elmeco da vico San Liborio al mondo, 2019.

59. F.D. Perillo, D. Bizjak, R.A. Tundo, The Company in the Backpack. Elmeco's, from Vico San Liborio to the World, 2019.

${ }^{x v i}$ Con scritti di Beatriz Acevedo, Omar Aktouf, Noortje van Amsterdam, Per Olof Berg, Davide Bizjak, Ilaria Boncori, Jo Brewis, Peter Case, Patricia Ehrensal, Ken Ehrensal, Hugo Gaggiotti, Silvia Gherardi, Claes Gustafsson, Campbell Jones, Nina Kivinen, Monika Kostera, Kristian J Kreiner, Thomas Taro Lennerfors, Marcus Lindahl, Steve Linstead, Tomek Ludwicki, Takashi Majima, Laura Mitchell, Albert J. Mills, Jean Helms Mills, Luc Peters, Alf Rehn, Harriet Shortt, Luigi Maria Sicca, Antonio Strati, Sam Warren, Robert Witkin, Anthony R. Yue, Peter Zackariasson.

${ }^{x v I I}$ Con scritti di Luigi Guatri, Giovanni Zanetti, Adriano Giannola, Franco Amatori, Renato Mele, Francesco Testa e Riccardo Mercurio; Enzo Rullani, Paolo de Vita, Salvatore Vicari, Luigi Cantone, Pierpaolo Testa e Vincenzo Basile; Marco Ferretti, Maria Rosaria Napolitano, Francesco Izzo, Enrico Bonetti, Antonio Capaldo, Paolo Calvosa, Davide Bizjak.

xvır Con scritti di Stefano De Luca e Guido Solza.

${ }^{x 1 x}$ Con scritti di Chiara Bombardieri, Paola Degli Esposti, Mariagabriella Fornasiero, Valentina Galloni, Alessandro Gazzotti, Claudia Giordani, Federica Guidi, Annalisa Managlia, Umberto Mossetti, Michele Recanatini, Elisa Schiavina, Stefania Spaggiari, Patrizia Tomba, Cinzia Vecchi. 
60. M. Castro Espín ${ }^{x x}$, Persone transessuali a Cuba (con prefazione di L.M. Sicca), 2019.

61. G. van Wulfen, La spedizione per innovare. Un kit di strumenti visual per far partire l'innovazione (I edizione italiana a cura di M.V. Colucci, A. Forciniti, L. Migliola, L. Rossi), 2019.

62. P. Senge ${ }^{x \times 1}$, La quinta disciplina: l'arte e la pratica dell'apprendimento organizzativo (ed. 2006), (edizione italiana a cura di L.M. Sicca), 2019.

63. V. Basile, Marketing Performance Measurement in Fmcg Share of Wallet in Retailing Industry (con prefazione di L. Cantone), 2020.

64. A. Moretti, R. Tabacco, I Menuzzo e CAME. Una storia di passione per il prodotto, $i$ clienti e le relazioni (con postfazione di R. Samiolo), 2020.

65. B. Masiello, Il lato oscuro delle reti. Strategie d'impresa e capitale sociale, (con introduzione di F. Izzo) 2020.

66. F. Izzo, B. Masiello, Le fabbriche bianche. I processi di innovazione nelle imprese creative, 2020.

67. D. Salvatore, Saperi manageriali, crescita sostenibile e sud interno. Quali contributi possono offrire gli studi di organizzazione aziendale per una crescita economica sostenibile?, 2020.

68. P. Canonico, Understanding the role of knowledge in project settings, 2020.

69. I. Boncori, T. Loughran ${ }^{x \times 1}$, Health and Wellbeing. The University of Essex Reader (con prefazione di A. Forster), 2020.

${ }^{x x}$ Con scritti di Mario Bottone e Paolo Valerio; Christian Ballarin; Davide Bizjak e Cristiano Scandurra; Rossella Bonito Oliva; Giuseppe Burgio; Porpora Marcasciano; Mary Nicotra; Alessandra Riccio.

xxi Con scritti di Luigi Maria Sicca, Edoardo Mollona e Stefano Armenia; Nicola Andreottola, Simona Capecelatro, Davide Coppola; Sergio Barile e Francesca Iandolo; Mariavittoria Cicellin e Stefano Consiglio; Corrado Cuccurullo; Davide Bizjak, Paolo Canonico e Vito Lasala; Marcello Martinez e Mario Pezzillo Iacono; Stefano Mazzoleni e Francesco Giannino; Mario Nicodemi; Francesco Perillo; Francesco Piro; Apollonia Striano.

xxII Con scritti di Anthony Forster; Ilaria Boncori and Tracey Loughran; Martyna Śliwa; Charlie V.L. Smith; Lauren O'Connell; Abby Shovlin; Peter J. Martin and Edward M. Holt; Nileema Vaswani and Ilaria Boncori; Filippo Sinicato; Jaime Lindsey; Ewen Speed, Susan McPherson and Peter Beresford; Francine Bailey; Sean Seeger. 
70. J. Altmanova, L. Cannavacciuolo, M. Ottaiano, K. Russo (a cura di) ${ }^{x \times I I}$, Across the University. Linguaggi, narrazioni, rappresentazioni del mondo accademico, 2020.

71. M.T. Como, La Sala degli Angeli nel complesso conventuale di Suor Orsola Benincasa. Per una storia della costruzione, (con prefazione di L. d'Alessandro), 2020.

72. S. Cardone, Breviario di Pedagogia Teatrale (con prefazione di A. Bergamo), 2020.

73. E. Sacerdote, Legendary brands. Valutazioni e riflessioni strategiche, 2020.

74. C. Bruno, Gestire l'industria dell'emozione. Un'analisi manageriale dell'attività dei teatri lirici (con prefazione di G. Fraquelli), 2020.

75. S. Consiglio e M. D'Isanto, La cultura che cambia (con premessa di E. D'Errico, prefazione di C. Borgomeo e postfazione di G. Volpe), 2020.

76. G. Nolfe, L.M. Sicca ${ }^{\text {xxrv }}$, Mobbing. Narrazioni individuali e organizzative (con prefazione di B. Czarniawska e postfazione di B. Poggio), 2020.

77. S. Armenia, The value of Systems Thinking and System Dynamics in the management of complex organizations. A selection of case studies (con prefazione di E. Mollona), 2020.

78. B. Czarniawska, Per una teori adell'organizzazione, edizione italiana a cura di L.M. Sicca, F. Piro, 2020

xxII Con scritti di Augusto Guarino, Maria Laudando, Maria Alessandra Giovannini, Giovanni Rotiroti, Ruth Amar, Valeria Sperti, Andrea Pezzè, Irma Carannante, Oriana Palusci, Antonio Saccone, Germana Volpe, Laura Cannavacciuolo, Giuseppina Notaro, Fabio Rodríguez Amaya, M. Cristina Lombardi, Marco Ottaiano, Franco Paris, Francesca De Cesare, Katherine Russo, Angela Buono, Anna Mongibello, Giovanni Agresti, Jana Altmanova, Giuliana Regnoli, Rosario Gallone, Vincenzo Bavaro, Federico Pio Gentile.

xxiv Con scritti di Giovanni Nolfe, Luigi Maria Sicca, Gemma Zontini, Christophe Dejours; Giuseppe Recinto, Raffaello Santagata de castro e Fabio Dell'Aversana, Mariapia Garavaglia; Luca Solari, Enzo Cordaro. 
Finito di stampare nel mese di dicembre 2020 dalla Vulcanica srl - Nola (NA) 
